\title{
Fuori da Atene. Miti e tradizioni su Oreste in Grecia antica. Prefazione di Manuela Giordano
}

Canterano: Aracne editrice, 2017. Pp. 389; ISBN 978-88-255-0071-4. €20

\section{Stefano Acerbo}

\section{(2) OpenEdition}

\section{Journals}

Edizione digitale

URL: http://journals.openedition.org/mythos/387

DOI: $10.4000 /$ mythos.387

ISSN: 2037-7746

\section{Editore}

Salvatore Sciascia Editore

\section{Edizione cartacea}

Data di pubblicazione: 1 dicembre 2018

Paginazione: 169-170

ISBN: 978-88-8241-501-3

ISSN: $1972-2516$

\section{Notizia bibliografica digitale}

Stefano Acerbo, «Fuori da Atene. Miti e tradizioni su Oreste in Grecia antica. Prefazione di Manuela Giordano », Mythos [Online], 12 | 2018, online dal 24 septembre 2019, consultato il 25 septembre 2020. URL : http://journals.openedition.org/mythos/387 ; DOI : https://doi.org/10.4000/mythos.387 


\section{Luca Pucci}

Fuori da Atene. Miti e tradizioni su Oreste in Grecia antica. Prefazione di Manuela Giordano.

Canterano: Aracne editrice, 2017. Pp. 389; ISBN 978-88-255-0071-4. €20.

Stefano Acerbo - Università di Pisa - acerboste@gmail.com

Il volume di Pucci offre una raccolta ed un'analisi delle notizie tramandate dalle fonti antiche relative alle tradizioni mitiche su Oreste che si situano al di fuori dell'Attica. La scelta dell'argomento non si spiega a partire da considerazioni di natura meramente geografica, ma implica una presa di posizione originale rispetto ai precedenti studi dedicati all'eroe. Essi hanno attribuito una posizione di rilievo alla versione delfico-ateniese del mito, che troverebbe la sua più pura espressione nell'Orestea di Eschilo, finendo per trascurare le altre tradizioni. L'autore ha, invece, il merito di porre in questione il carattere secondario delle notizie che situano la vicenda di Oreste all'interno di un piano geografico e ideologico diverso da quello eschileo. In tal modo, giunge a ricostruire percorsi diacronici che rivelano significati e rappresentazioni diverse da quelle note dalla scena drammatica ateniese, ma, non per questo, deteriori.

Per giungere a simili risultati, si serve di una analisi storico-filologica delle fonti antiche estremamente rigorosa e, al contempo, degli strumenti offerti dall'antropologia. Alla esplicitazione di tali strumenti è dedicato il primo capitolo del volume, che si avvale di una bibliografia aggiornata. II mito è definito a partire da un approccio socio-costruttivista. L'attenzione prestata al contesto sociale e culturale cui riferiscono i racconti mitici permette di offrire una chiara disamina della spinosa questione del rapporto tra essi e il rito. La seconda parte del capitolo si sofferma sui temi che caratterizzano il complesso mitico relativo a Oreste, vale a dire vendetta, contaminazione e purificazione.

Proprio il tema della vendetta offre il filo conduttore all'interno di un volume organizzato secondo un principio geografico. Sono analizzate le tradizioni relative alle vicende di Oreste in Argolide, in Laconia, Arcadia e, infine, nelle altre regioni. La ricerca non ha l'illusione di ritrovare un nucleo epicorico originario da cui si sarebbero generate le altre tradizioni: la pluralità spaziale è, piuttosto, un elemento che caratterizza l'eroe perché, come osservato nelle conclusioni, questi non ha una chiara identità regionale e, allo stesso tempo, non è una figura panellenica come il padre. La pluralità di soluzioni mostrate dalle diverse fonti è, piuttosto, interpretata a partire dalla categoria della polivalenza delle immagini, che riconosce come una stessa immagine mitica possa veicolare valori differenti in diversi contesti.

Le notizie collocate in Argolide risalgono già a Omero, cui è dedicata la prima sezione del secondo capitolo. L'autore rileva la traccia di diverse tradizioni, che si spiega in ragione della pluralità spaziale e temporale dei contesti di esecuzione dei poemi. II poeta avrebbe privilegiato una tradizione che, dirigendo l'azione di Oreste solo contro Egisto, permetteva di tacere l'uccisione di Clitemnestra, privando, così, di problematicità il tema della vendetta. A un ambito argolico sono ricondotte anche le due versioni ricordate da Euripide nell'Elettra e nell'Oreste. L'analisi in questo caso si sposta su aspetti inerenti al diritto e ai problemi morali e filosofici posti dal dibattito sulla vendetta.

II terzo capitolo è dedicato alla Laconia, in cui sono attestati molti racconti relativi alle vicende di Oreste. L'autore organizza l'analisi secondo un'ulteriore suddivisione geografica, 
riconducendo le notizie ai diversi centri della regione. La scelta è dettata dalla volontà di contrastare la tendenza a trascurare le differenze tra la localizzazione ad Amiclea, presente in Pindaro, e quella spartana, ricordata da altri autori. Nel primo caso, le tradizioni su Oreste sono interpretate a partire dal contesto pragmatico degli epinici e dai rapporti che le tradizioni mitiche instaurano tra questo centro e Tebe. A un ambito spartano sono, invece, ricondotte le notizie sulla funzione di ecista di Oreste, le quali sembrano appartenere a una tradizione alternativa a quelle del suo esilio o del suo ritorno in patria. La fondazione di Tenedo testimonia i rapporti tra Laconia e Eolide d'Asia. Nell'interpretazione di Pucci, tale viaggio mantiene, comunque, una funzione espiatrice ed è accostato alla pratica, che si ritrova in vari contesti coloniali, di sacrificare ad Apollo la decima. II capitolo si conclude con uno studio del racconto erodoteo sulla traslazione a Sparta delle ossa dell'eroe.

II quarto capitolo si concentra sulla presenza dell'eroe in Arcadia. Tra l'opzione di un Oreste originariamente arcadico, e quella di un'appropriazione epicorica della saga degli Atridi, Pucci sceglie di tentare una nuova via di ricerca, evidenziando come questa regione fosse nell'immaginario greco una terra privilegiata per la supplica e la purificazione. Tali tradizioni risalirebbero, comunque, a un'epoca più antica rispetto alla tragedia attica, come mostrerebbe il racconto dell'autofagia del dito nella piana di Megalopoli. In esso è riconosciuto un nucleo databile al VII e al VI secolo, che sarebbe stato aggiornato e rivisto nel $\mathrm{V}$, per renderlo compatibile con la tradizione delfico-ateniese. A rappresentazioni precedenti a quelle di Eschilo rinvierebbe anche la notizia di Ferecide, secondo cui l'eroe sarebbe giunto presso un santuario di Artemide in Arcadia e avrebbe dato il suo nome alla città di Orasterion. L'autore mostra una notevole attenzione filologica alla difficile ricostruzione del discorso attribuibile a Ferecide a partire dalla testimonianza degli scoli. Sempre in Arcadia sarebbe avvenuta la morte, come già implicito nella notizia erodotea della traslazione delle sue ossa da Tegea, che trova conferma in uno scolio che cita Asclepiade di Tragilo e nella Biblioteca dello ps. Apollodoro, che, però, indica in Oresteion il luogo in cui l'eroe sarebbe stato morso mortalmente da un serpente.

Il libro è chiuso da un capitolo in cui sono esaminate notizie collocate in altri luoghi e, in particolare, il soggiorno dell'eroe in Elide presso Strofio e il sacrificio rituale a Cerinea. Questi nuclei mitici possono conservare tracce di tradizioni autonome rispetto alla versione delfico-ateniese.

Tutto il volume è caratterizzato da una costante attenzione al rapporto con le forme giuridiche e i riti religiosi e purificatori. In tal modo, l'autore illumina le forme di pensiero e le realtà sociali che hanno contribuito a plasmare i racconti su Oreste, tracciando una possibile via per studiare il rapporto tra mito e storia. Molti paragrafi costituiscono, inoltre, uno scrupoloso tentativo di ricostruzione delle tradizioni mitiche e dei loro contesti di esecuzione: l'autore raccoglie una impressionante mole di dati, proponendo interpretazioni originali e puntuali che rendono quest'opera un vero e proprio "reference work" sull'argomento. 